\title{
Target acquisition and acute promyelocytic leukemia
}

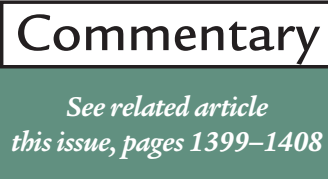

\author{
David A. Egan and Ronald M. Evans \\ Howard Hughes Medical Institute, The Salk Institute for Biological Studies, 10010 North Torrey Pines Road, La Jolla, California 92037, USA. \\ Phone: (619) 453-4100 ext. 1302; Fax: (619) 455-1349; E-mail: evans@salk.edu.
}

Acute promyelocytic leukemia (APL) is characterized by a variety of chromosomal translocations into the retinoic acid receptor- $\alpha$ (RAR $\alpha)$ gene. The first of these cloned was $t(15 ; 17)$, a translocation that results in fusion of the PML gene with RAR $\alpha$. This translocation is also the most prevalent, found in $95 \%$ of APL cases. Other translocations associated with fusion proteins in variant APLs were subsequently discovered: $\mathrm{t}(11 ; 17)$ PLZF-RAR $\alpha$ (found in less than $5 \%$ of cases), another $\mathrm{t}(11 ; 17)$ translocation, NuMA-RAR $\alpha$ (less than $1 \%$ ), and $t(5 ; 17)$ NPM-RAR $\alpha$ (less than 1\%). All of these, with the exception of the PLZF-RAR $\alpha$ translocation, are associated with a disease that responds to high doses of retinoids (1). The realization that all of these translocations involved RAR $\alpha$ led to the proposal that the disease is caused by dysfunctional regulation of retinoidinducible genes critical to myeloid differentiation. Recent work supporting this idea has shown that PML-RAR $\alpha$ has a reduced ability to release transcriptional corepressors such as SMRT and NCoR in response to retinoids and that the PLZF portion of the PLZF-RAR $\alpha$ fusion constitutively binds these repressors $(2,3)$. This would suggest that in APL cells, RAR $\alpha$ target genes may be transcriptionally super-repressed, and may also explain why PML-RAR $\alpha$ APL responds to high doses of retinoids, whereas PLZF-RAR $\alpha$ APL is retinoid resistant. The ultimate proof of the hypothesis, however, has awaited the identification of critical target genes, the regulation of which is disrupted by the fusion proteins. In this issue of the JCI, Park et al. propose that the gene for the myeloid-specific transcription factor C/EBPE may be such a target (4).

Previous studies have shown that C/EBPE transcription is induced by retinoids during granulocytic, but not monocytic, differentiation (5). Mice in which the gene is deleted exhibit impaired granulopoiesis (6). Therefore C/EBPE has many of the predicted characteristics of a critical target gene in APL. It remained to be demonstrated that its promoter contained a functional retinoic acid response element (RARE) and that overexpression of $\mathrm{C} / \mathrm{EBP} \varepsilon$ alone was sufficient to induce granulocytic differentiation. These key experiments are presented in this issue.

The authors identified a RARE in the $\mathrm{C} / \mathrm{EBP} \varepsilon$ promoter and showed it to be functional in transfection assays. They demonstrated that C/EBPE expression could be induced in a ligand-dependent manner by PML-RAR $\alpha$ (a fusion product found in a retinoid-sensitive APL) but not by PLZF-RAR $\alpha$ (a fusion product found in a retinoid-resistant APL). Overexpression of C/EBPE in U937 cells was then shown to be sufficient for the induction of granulopoiesis, as evidenced by morphological changes and an increase in the expression of granulocytic markers such as CD11b, CD66b, lactoferrin, and collagenase. The expected decrease in proliferation and increase in apoptosis were also observed. Therefore, the authors are justified in proposing that C/EBPE may be an important downstream target of RAR $\alpha$ in myeloid differentiation.

What makes C/EBPE especially intriguing in this role are the findings, outlined in an earlier paper from the Koeffler laboratory, that suggest that $\mathrm{C} / \mathrm{EBP \varepsilon}$ expression may be a node at which cross-talk between regulatory pathways occurs. The differentiation agents DMSO and HMBA were shown to induce C/EBPE mRNA in the NB4 APL cell line, but only after the cells were primed by a transient 30-minute incubation with retinoic acid (7). The characterization of the pathways that cooperate with retinoids in the induction of $\mathrm{C} / \mathrm{EBP} \varepsilon$, and indeed independent pathways that regulate C/EBPE expression or function, will now be a priority. Knowledge of such pathways may lead to the development of therapeutic strategies whereby C/EBPE transcription can be induced in the absence of retinoids. These strategies might be especially useful for the treatment of retinoid-resist- ant APLs, such those resulting from the $\mathrm{t}(11 ; 17)$ PLZF-RAR $\alpha$ translocation, or APLs in which retinoid resistance is mediated by mutations in the PMLRAR $\alpha$ ligand-binding domain. It will be a challenging task, however, to unravel the complexities of the gene's regulation. It is already known, for example, that the C/EBPE gene can be transcribed from alternate promoters (8).

Identification of the genes regulated by $\mathrm{C} / \mathrm{EPB} \varepsilon$ will give a more complete understanding of its role in myeloid differentiation; the application of recently developed DNA array technologies may accelerate this process. $\mathrm{C} / \mathrm{EBPE}$ is a member of a growing family of transcription factors; in addition to C/EBPE, at least 3 of these factors (C/EBP $\alpha, \beta$, and $\delta$ ) are expressed during hematopoiesis (9). The various $\mathrm{C} / \mathrm{EBP}$ family members are known to be capable of both homo- and heterodimerization, and because the

\section{They demonstrated that C/EBPE could be induced in a ligand-dependent manner by $P M L-R A R \alpha$. . but not by PLZF-RAR $\alpha$. .}

expression of $\mathrm{C} / \mathrm{EBP} \alpha$ and $\mathrm{C} / \mathrm{EBP \varepsilon}$ are known to overlap, it is probable that they functionally interact during myeloid differentiation. This will make an analysis of $\mathrm{C} / \mathrm{EBP} \varepsilon$ target gene regulation quite challenging. To make matters even more complicated, the C/EBPE gene, through the use of alternative splicing and alternative promoters, encodes 3 different proteins. All of these have identical dimerization and DNA-binding domains, but different $\mathrm{NH}_{2}$-terminal regions. The functional significance of these differences is unknown (8).

Is the suppression of C/EBPE gene expression the common theme in all APL variants? One experiment presented by Park et al. would suggest that it is not. The overexpression of PLZF-RAR $\alpha$ 
in U937 cells in the absence of retinoids did not repress C/EBPE expression, whereas the overexpression of PMLRAR $\alpha$ did. This would suggest that PLZF-RAR $\alpha$ and PML-RAR $\alpha$ might act through different pathways, with different target specificity.

Now that we are beginning to identify downstream targets of the retinoid receptors, we can look forward to a great increase in our understanding of the molecular basis of myeloid differentiation. This, allied with recent studies that have shed light on the function of PML in cell growth, apoptosis, and transcriptional regulation (1), should pave the way for a deeper understanding of the molecular events that are involved in the development of APL.

1. Lin, R.J., Egan, D.A., and Evans, R.M. 1999. Molecular genetics of acute promyelocytic leukemia. Trends Genet. 15:179-184.

2. Lin, R.J., et al. 1998. Role of the histone deacetylase complex in acute promyelocytic leukaemia. Nature. 391:811-814.

3. Grignani, F., et al. 1998. Fusion proteins of the retinoic acid receptor-alpha recruit histone deacetylase in promyelocytic leukaemia. Nature. 391:815-818.

4. Park, D.J., et al. 1999. CCAAT/enhancer binding protein $\varepsilon$ is a potential retinoid target gene in acute promyelocytic leukemia treatment. J. Clin. Invest. 103:1399-1408.

5. Morosetti, R., et al. 1997. A novel myeloid transcription factor, C/EBP epsilon, is upregulated during granulocytic, but not monocytic, differentiation. Blood. 90:2591-2600.

6. Yamanaka, R, et al. 1997. Impaired granulopoiesis, myelodysplasia, and early lethality in CCAAT/enhancer binding protein epsilon-deficient mice. Proc. Natl. Acad. Sci. USA. 94:13187-13192.

7. Chih, D.Y., Chumakov, A.M., Park, D.J., Silla, A.G., and Koeffler, H.P. 1997. Modulation of mRNA expression of a novel human myeloid-selective CCAAT/enhancer binding protein gene (C/EBP epsilon). Blood. 90:2987-2994.

8. Yamanaka, R., et al. 1991. CCAAT/enhancer binding protein epsilon is preferentially up-regulated during granulocytic differentiation and its functional versatility is determined by alternative use of promoters and differential splicing. Proc. Natl. Acad. Sci. USA. 94:6462-6467.

9. Dhordain, P., et al. 1997. Corepressor SMRT binds the $\mathrm{BTB} / \mathrm{POZ}$ repressing domain of the LAZ3/BCL6 oncoprotein. Proc. Natl. Acad. Sci. USA. 94:10762-10767. 